\section{Hypoxia modulates the purine salvage pathway and decreases red blood cell and supernatant levels of hypoxanthine during refrigerated storage}

\author{
Travis Nemkov, ${ }^{1 \#}$ Kaiqi Sun, ${ }^{2 \#}$ Julie A. Reisz, ${ }^{1 \#}$ Anren Song, ${ }^{2}$ Tatsuro Yoshida, ${ }^{3}$ \\ Andrew Dunham, ${ }^{3}$ Matthew J. Wither, ${ }^{1}$ Richard O. Francis, ${ }^{4}$ Robert C. Roach, ${ }^{5}$ \\ Monika Dzieciatkowska, ${ }^{1}$ Stephen C. Rogers, ${ }^{6}$ Allan Doctor, ${ }^{6}$ Anastasios \\ Kriebardis, ${ }^{7}$ Marianna Antonelou, ${ }^{8}$ Issidora Papassideri, ${ }^{8}$ Carolyn T. Young, ${ }^{9}$ \\ Tiffany A. Thomas, ${ }^{4}$ Kirk C. Hansen, ${ }^{1}$ Steven L. Spitalnik, ${ }^{4}$ Yang Xia, ${ }^{2}$ James C. \\ Zimring, ${ }^{10}$ Eldad A. Hod ${ }^{4}$ and Angelo D'Alessandro ${ }^{1,11}$
}

\begin{abstract}
${ }^{1}$ Department of Biochemistry and Molecular Genetics, University of Colorado Denver Anschutz Medical Campus, Aurora, CO, USA; ${ }^{2}$ Department of Biochemistry, University of Texas Houston - School of Medicine, Houston, TX, USA; ${ }^{3}$ New Health Sciences Inc, Boston, MA, USA; ${ }^{4}$ Department of Pathology \& Cell Biology, Columbia University Medical Center, New York, NY, USA; ${ }^{5}$ Altitude Research Center, University of Colorado Denver Anschutz Medical Campus, Aurora, CO, USA; ${ }^{6}$ Division of Critical Care Medicine, Department of Pediatrics, School of Medicine, Washington University in St Louis, St Louis, MO, USA; ' ${ }^{7}$ epartment of Medical Laboratories, Technological and Educational Institute of Athens, Greece; ${ }^{8}$ Department of Biology, National and Kapodistrian University of Athens, Greece; ${ }^{9}$ Rhode Island Blood Center, Providence, RI, USA and ${ }^{10}$ BloodWorks Northwest, Seattle, WA, USA. ${ }^{11}$ Boettcher Investigator.
\end{abstract}

${ }^{\# T N}, K S$ and JAR contributed equally to this work and share the first authorship.
H ypoxanthine catabolism in vivo is potentially dangerous as it fuels production of urate and, most importantly, hydrogen peroxide. However, it is unclear whether accumulation of intracellular and supernatant hypoxanthine in stored red blood cell units is clinically relevant for transfused recipients. Leukoreduced red blood cells from glucose-6-phosphate dehydrogenase-normal or -deficient human volunteers were stored in AS-3 under normoxic, hyperoxic, or hypoxic conditions (with oxygen saturation ranging from $<3 \%$ to $>95 \%$ ). Red blood cells from healthy human volunteers were also collected at sea level or after 1-7 days at high altitude (>5000 m). Finally, C57BL/6J mouse red blood cells were incubated in vitro with ${ }^{13} \mathrm{C}_{1}$-aspartate or ${ }^{13} \mathrm{C}_{5}$-adenosine under normoxic or hypoxic conditions, with or without deoxycoformycin, a purine deaminase inhibitor. Metabolomics analyses were performed on human and mouse red blood cells stored for up to 42 or 14 days, respectively, and correlated with $24 \mathrm{~h}$ post-transfusion red blood cell recovery. Hypoxanthine increased in stored red blood cell units as a function of oxygen levels. Stored red blood cells from human glucose-6-phosphate dehydrogenase-deficient donors had higher levels of deaminated purines. Hypoxia in vitro and in vivo decreased purine oxidation and enhanced purine salvage reactions in human and mouse red blood cells, which was partly explained by decreased adenosine monophosphate deaminase activity. In addition, hypoxanthine levels negatively correlated with post-transfusion red blood cell recovery in mice and - preliminarily albeit significantly - in humans. In conclusion, hypoxanthine is an in vitro metabolic marker of the red blood cell storage lesion that negatively correlates with post-transfusion recovery in vivo. Storage-dependent hypoxanthine accumulation is ameliorated by hypoxia-induced decreases in purine deamination reaction rates.

\section{Correspondence:}

angelo.dalessandro@ucdenver.edu

Received: August 11, 2017.

Accepted: October 24, 2017.

Pre-published: October 27, 2017.

doi:10.3324/haematol.2017.178608

Check the online version for the most updated information on this article, online supplements, and information on authorship \& disclosures: www.haematologica.org/content/103/2/361

\section{(C)2018 Ferrata Storti Foundation}

Material published in Haematologica is covered by copyright. All rights are reserved to the Ferrata Storti Foundation. Use of published material is allowed under the following terms and conditions:

https://creativecommons.org/licenses/by-nc/4.0/legalcode. Copies of published material are allowed for personal or internal use. Sharing published material for non-commercial purposes is subject to the following conditions:

https://creativecommons.org//icenses/by-nc/4.0/legalcode, sect. 3. Reproducing and sharing published material for commercial purposes is not allowed without permission in writing from the publisher. 


\section{Introduction}

Packed red blood cell (RBC) transfusions are life-saving interventions for millions of recipients every year $(\sim 11.3$ million units transfused/year in the USA alone $)^{1}$. Refrigerated RBC storage is required to make the 100 million units collected worldwide every year available for transfusion. However, refrigerated RBC storage induces many biochemical and morphological alterations, collectively termed the "storage lesion." ${ }^{2-5}$ Some alterations are promoted by oxidative stress, arising within the first 2 weeks of storage,,$^{6,7}$ targeting proteins, ${ }^{8-11}$ lipids, ${ }^{12-15}$ and various small molecules, including purines. . $^{1-19}$ These observations, together with early depletion of high energy phosphate compounds, including adenosine triphosphate (ATP) and 2,3-diphosphoglycerate (2,3-DPG), prompted many to question whether the storage lesion compromises RBC transfusion safety and efficacy.

Despite evidence documenting the evolution and severity of the storage lesion, ${ }^{2-4}$ whether prolonged RBC storage duration adversely affects transfused recipients is debatable. Recent randomized clinical trials concluded that the general standard of care would not be improved by exclusively issuing fresh RBC, at least for the studied clinical indications and within the statistical power of these studies. ${ }^{20}$ However, these clinical trials did not examine blood that was particularly old (e.g., $>35$ days). Recent evidence indicates that the storage lesion does not develop linearly and only transfusing $>35$-day old RBC induces increases in circulating non-transferrin-bound iron in healthy recipients. ${ }^{21}$ In parallel, increases in adverse outcomes have been observed in high-risk patients receiving blood units aged $>35$ days. ${ }^{22}$ Thus, to the extent that in vivo hemolysis and non-transferrin-bound iron are mediators of the adverse effects of transfusion, the clinical trials to date have not shown that transfusing old blood is safe.

Current storage solutions make it possible to store RBC for up to 42 days with an average $\sim 17 \%$ loss of transfusion potency at outdate ${ }^{23}$ based on ${ }^{51} \mathrm{Cr}$-labeled post-transfusion recovery (PTR) studies in healthy human volunteers ${ }^{24}$ which provide information on the ability of RBC to circulate, but not necessarily their ability to deliver oxygen. This is relevant when considering the effects of the loss of potency in massively transfused recipients, such as trauma patients. ${ }^{23}$

Despite reassuring evidence from clinical trials, further improvement in RBC storage strategies are possible, as recommended by the US National Heart, Lung, and Blood Institute..$^{25}$ To this end, advances in the molecular understanding of the storage lesion have fostered the design of novel storage solutions (e.g., alkaline additives ${ }^{26}$ ) and strategies (e.g., hypoxic storage ${ }^{27}$ ) to improve storage quality. In parallel, recently identified "omics" markers of storage age $e^{28-30}$ may prove useful for benchmarking potential improvements in storage quality, once their association with post-transfusion outcomes has been clearly demonstrated. The present study addresses this by focusing on hypoxanthine, ${ }^{17,28,31}$ a deaminated purine resulting from the metabolism of ATP, adenosine monophosphate (AMP), and adenosine in mature RBC. Recently, Casali et al., ${ }^{31}$ Bordbar et al..$^{16}$ and Paglia et al. ${ }^{28}$ reported that end-of-storage SAGM RBC and supernatants are characterized by levels of hypoxanthine as high as $\sim 450$ and $1000 \mu \mathrm{M}$, respectively. As previously noted,$^{31}$ under physiological conditions the concentration of hypoxanthine is very low, both inside erythrocytes ( $9.3 \mathrm{nM})$ and in human plasma $(1-8 \mu \mathrm{M})$. Transfusion of a single unit of end-of-storage blood containing almost millimolar levels of hypoxanthine could thus result in circulating hypoxanthine levels of $\sim 100 \mu \mathrm{M}$, an amount that would further increase proportionally to the number of older units transfused to the recipient. Notably, concentrations as high as $100 \mu \mathrm{M}$ are used in cytotoxicity assays to produce toxic amounts of hydrogen peroxide generated through the xanthine dehydrogenase/oxidase-catalyzed conversion of hypoxanthine to urate. ${ }^{31}$

On this background, in the present study, we provide absolute quantitative measurements of hypoxanthine in cells and supernatants of AS-3 packed RBC. We report negative correlations between RBC hypoxanthine levels in vitro and PTR in vivo in 14 different mouse strains and, preliminarily, in healthy human volunteers, indicating the potential clinical relevance of this metabolic lesion. We also provide a possible mechanistic explanation regarding the role of AMP deaminase (AMPD) activation in human and mouse RBC as a function of hemoglobin oxygen saturation $\left(\mathrm{SO}_{2}\right)$ and resulting oxidative stress in vitro and in vivo. Finally, by combining state-of-the-art deep proteomics, quantitative mass spectrometry (MS), tracing experiments with stable isotope-labeled substrates, and pharmacological inhibition of purine deaminases, we identify the role of hypoxia in preventing AMPD activation, thereby decreasing the storage-dependent accumulation of deaminated purines, particularly hypoxanthine.

\section{Methods}

Blood samples were collected from healthy donor volunteers upon receiving written informed consent and in conformity with the Declaration of Helsinki under the protocol approved by the relative institutions, including the University of Texas Houston and University of Colorado Denver institutional review boards (n. AWC-14-0127 and 11-1581, respectively). Commercial reagents were purchased from Sigma-Aldrich (Saint Louis, MO, USA) unless otherwise noted.

\section{Glucose-6-phosphate dehydrogenase-normal and -deficient human red blood cells, stored under normoxic or hypoxic conditions}

Blood was collected from healthy glucose-6-phosphate dehydrogenase (G6PD)-normal donors at the Bonfils Blood Center (Denver, CO, USA) or from G6PD-deficient donors (Mediterranean variant) in Athens (Greece) according to the Declaration of Helsinki. Filter leukocyte-reduced (Pall Medical, Braintree, MA, USA) packed RBC were stored in CP2D-AS-3 ( $n=4$; Haemonetics Corp., Braintree, MA, USA) or CPD-SAGM ( $\mathrm{n}=6)$. Units were sampled in a sterile manner (15 mL per time point) on days $0,21,42$, and cells and supernatants were separated by centrifugation at $2000 \times \mathrm{g}$ for $10 \min$ at $4^{\circ} \mathrm{C}$.

\section{Mouse red blood cell storage under normoxic and hypoxic conditions with an adenosine monophosphate deaminase inhibitor}

$\mathrm{RBC}$ were collected aseptically by exsanguination from C57BL/6 mice (pool of $n=5$ per group) and stored for 14 days $^{32}$ in CPD-AS-3 under normoxic or hypoxic conditions $\left(\mathrm{O}_{2}=21 \%\right.$ or $8 \%$, respectively $)$, in the presence or absence 
of ${ }^{13} \mathrm{C}_{5}$-adenosine $(5 \mu \mathrm{M})$ and deoxycoformycin $(500 \mu \mathrm{M})$, an AMPD inhibitor $(500 \mu \mathrm{M})$, as described. ${ }^{33}$

\section{Post-transfusion recovery studies in healthy human donor volunteers}

PTR studies were performed at Columbia University Medical Center-New York Presbyterian Hospital in healthy volunteers receiving autologous packed $\mathrm{RBC}(\mathrm{n}=52)$, and were previously published ${ }^{21}$ without accompanying metabolomics data. Briefly, immediately before issue, a 25 $\mathrm{mL}$ sample of blood, obtained from the unit using a sterile docking device, was radiolabeled with ${ }^{51} \mathrm{Cr},{ }^{21}$ while a matching $500 \mu \mathrm{L}$ sample was immediately frozen for metabolomics analyses. At 1-4 $\mathrm{h}$ after transfusion of the unit, the ${ }^{51} \mathrm{Cr}$-labeled RBC sample was infused over $1 \mathrm{~min}$. Blood specimens were then obtained every $2.5 \mathrm{~min}$ between 5 and $15 \mathrm{~min}$ after infusion and used to extrapolate time zero and the final time point to calculate PTR. ${ }^{21}$ Hypoxanthine levels were measured in the transfusates of the subjects in the previously published study. ${ }^{21}$

\section{Post-transfusion recovery studies in mice}

The PTR studies in mice were performed as described previously, ${ }^{34}$ using multiple strains from Jackson Labs (Bar Harbor, ME, USA): KK/HIJ, LG/J, AKR/J, FVB/NJ, C3H/HeJ, DBA/2J, NOD/ShiLtJ, 129X1/SvJ, 129S1/SvImJ, A/J, BTBR $\mathrm{T}+\mathrm{tf} / \mathrm{J}, \mathrm{Balb} / \mathrm{cByJ}, \mathrm{C} 57 \mathrm{Bl} / 6 \mathrm{~J}$. UbiC-GFP male mice, on a C57BL/6 background, were bred to FVB/NJ females in the Bloodworks NW Research Institute Vivarium (Seattle, WA, USA) and offspring were used as transfusion recipients at 24-28 weeks of age. ${ }^{34}$

\section{Human red blood cell oxygen saturation 8 hours after donation}

CP2D-AS-3 RBC with controlled $\mathrm{SO}_{2}$ [i.e., $>95 \%$ (hyperoxic) to $<3 \%$ (hypoxic)] on day 0 were prepared in vented chambers (Difco BLL, Detroit, MI, USA). $\mathrm{SO}_{2}$ levels in 977 RBC units at the Rhode Island Blood Center were determined within $8 \mathrm{~h}$ of donation and routine processing (i.e. leukofiltration and storage in AS-3 under standard normoxic blood bank conditions), with methods previously described and validated. ${ }^{35}$

\section{High altitude studies}

$\mathrm{RBC}$ were collected from 12 male and nine female healthy human volunteers at sea level or after $3 \mathrm{~h}$ (ALT1 $\mathrm{am}),>8 \mathrm{~h}$ (ALT1 pm), or 7 days (ALT7) of exposure to high altitude hypoxia $(5260 \mathrm{~m}$ ) in Mt. Chacaltaya, Bolivia, within the framework of the AltitudeOmics study. ${ }^{36}$

\section{Red blood cell treatment with xanthine dehydrogenase/oxidase and hypoxanthine}

Human RBC were exposed to $1.5 \mathrm{mM}$ hypoxanthine in the presence of xanthine oxidase $(0.8 \mathrm{U} / \mathrm{mL})$ at $37^{\circ} \mathrm{C}$ for up to $6 \mathrm{~h}$ in a shaking water bath, as reported elsewhere. ${ }^{37}$

\section{Proteomic analyses}

Leukocyte-reduced human RBC from healthy donor volunteers were washed five times in phosphate-buffered saline prior to lysis in distilled water with sonication. Proteomic analyses of RBC membranes and cytosols were performed as described elsewhere ${ }^{38}$ using $30 \mu \mathrm{g}$ of protein per time point and a $4-12 \%$ gradient SDS-PAGE gel..$^{39}$ Bands were reduced, alkylated, trypsin digested, and then analyzed by nanoUHPLC-MS/MS (nanoEasy LC II coupled to a Q Exactive HF - Thermo Fisher, Bremen, Germany). Alternatively, RBC cytosolic proteins were depleted of hemoglobin using Hemovoid ${ }^{\mathrm{TM}}$ (Biotech support group, Monmouth Junction, NJ, USA), prior to high-pH reversed phase fractionation, ${ }^{40} 64$ fractions were collected (32 each for cytosol and membranes) over a $3 \mathrm{~h}$ gradient, prior to nano-UHPLC-MS/MS proteomics, as described previously. ${ }^{9}$ Error tolerant searches were performed using Mascot (v. 2.4) against the human UniprotKB database (release date 2015.1.8), including decoy sequences (cysteine carbamidomethylation and methionine oxidation set as fixed and variable modifications, respectively). Mass tolerances for membrane and vesicle data were set to $\pm 15 \mathrm{ppm}$ for precursor ions and $\pm 0.01 \mathrm{Da}$ for fragment ions. For all Mascot search results, peptide spectral matches were filtered at a $95 \%$ confidence threshold (excluding matches with an expectation value $>0.05$ )

\section{Sample processing and metabolite extraction}

RBC were separated by centrifugation $\left(10 \mathrm{~min}\right.$ at $4^{\circ} \mathrm{C}$ and $2500 \mathrm{xg}$ ) and then $50 \mu \mathrm{L}$ were extracted in $450 \mu \mathrm{L}$ of lysis buffer (methanol:acetonitrile:water 5:3:2) via ice cold extraction by vortexing for $30 \mathrm{~min}$ at $4^{\circ} \mathrm{C} \cdot \cdot^{14,41}$ Insoluble proteins were pelleted by centrifugation (10 min at $4^{\circ} \mathrm{C}$ and $10,000 \mathrm{xg}$ ) and supernatants were collected and stored at $-80^{\circ} \mathrm{C}$ until analysis.

\section{Ultrahigh performance liquid chromatography - mass spectrometry metabolomics}

Analyses were performed using a Vanquish UHPLC system coupled online to a $Q$ Exactive mass spectrometer (Thermo Fisher, Bremen, Germany). Samples were resolved over a Kinetex C18 column (2.1 x $150 \mathrm{~mm}, 1.7 \mu \mathrm{m}$; Phenomenex, Torrance, CA, USA) at $25^{\circ} \mathrm{C}$ using a $3 \mathrm{~min}$ isocratic condition of $5 \%$ acetonitrile, $95 \%$ water, and $0.1 \%$ formic acid flowing at $250 \mu \mathrm{L} / \mathrm{min},{ }^{42}$ or using a 9 min gradient at $400 \mu \mathrm{L} / \mathrm{min}$ from $5-95 \%$ B (A: water $/ 0.1 \%$ formic acid; B: acetonitrile $/ 0.1 \%$ formic acid). ${ }^{14} \mathrm{MS}$ analysis and data processing were performed as described elsewhere. ${ }^{14}$ Metabolite assignments, absolute quantification against heavy labeled internal standards and calibration curves of external standards (Cambridge Isotopes, Tewksbury, MA, USA), isotopologue distributions, and correction for expected natural abundances of ${ }^{13} \mathrm{C}$ isotopes were performed using MAVEN (Princeton, NJ, USA), as already described. ${ }^{42}$ Absolute quantities of hypoxanthine were determined using the following formula:

$$
\begin{gathered}
\text { Hypoxanthine }_{\text {Absoluteauant }}=(\text { Integrated Peak Area } \\
\text { Integrated Peak Area }_{\text {Heary }} / \\
\text { where } 10 \text { is the dilution factor }
\end{gathered}
$$

Graphs and statistical analyses ( $t$-test, repeated measures ANOVA, or Spearman correlation) were prepared with GraphPad Prism 5.0 (GraphPad Software, Inc., La Jolla, CA, USA).

\section{Results}

Hypoxanthine accumulates during murine and human red blood cell storage and negatively correlates with post-transfusion recovery

Intracellular and supernatant hypoxanthine levels progressively increase during standard storage of human RBC in AS-3 (Figure 1A), reaching concentrations as high as 450 

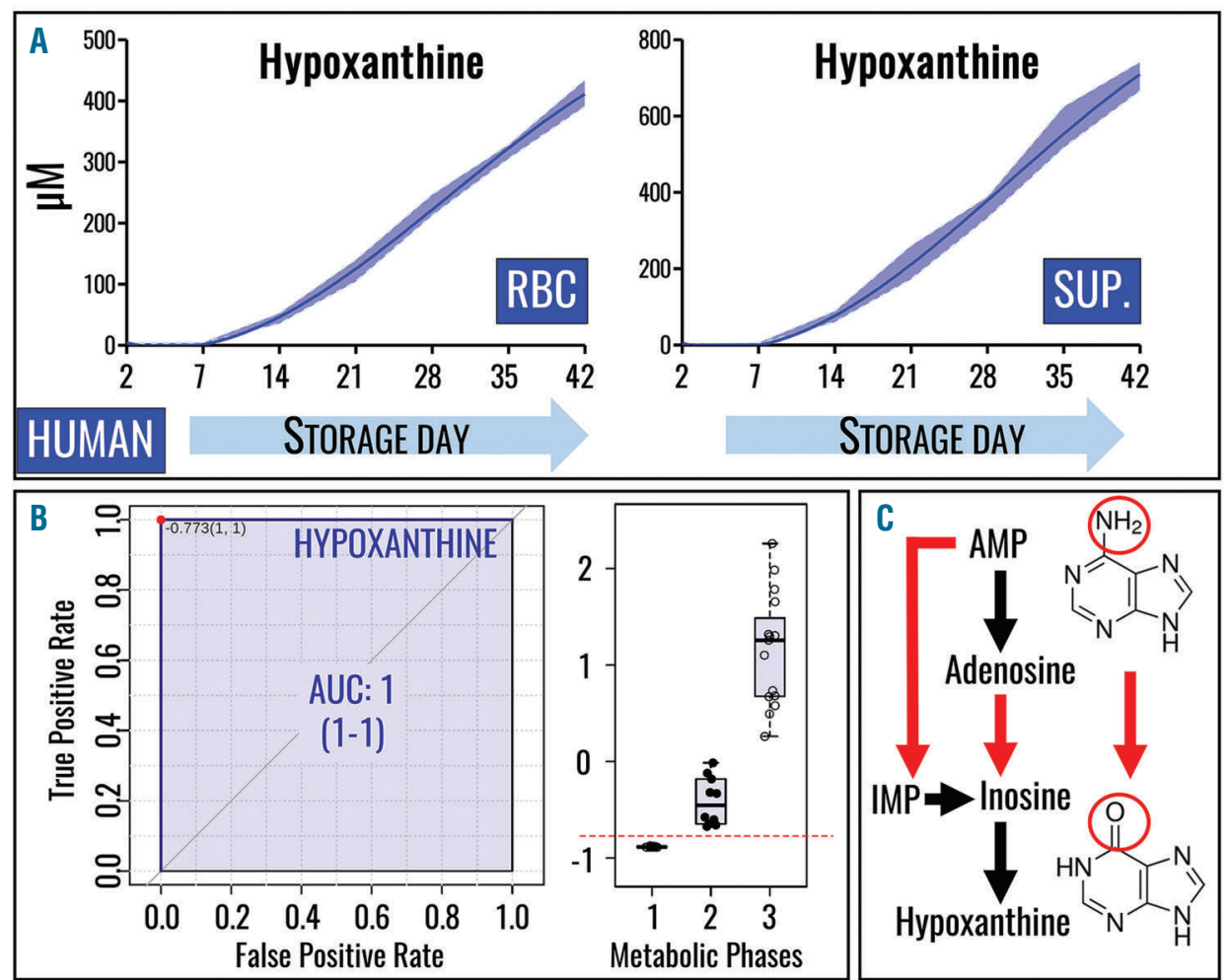

Figure 1. Hypoxanthine is a metabolic marker of the red blood cell storage lesion. (A) Intracellular (left) and supernatant (right) levels of hypoxanthine increase in packed RBC stored in the presence of AS-3. All data points shown on the $x$ axes were tested and interpolated with third order polynomial curves (not assuming linear evolution of hypoxanthine accumulation during storage) and median \pm ranges $(n=4)$ are shown (dark blue lines and light blue areas, respectively). (B) ROC curves identify hypoxanthine as a biomarker of the three metabolic stages of stored RBC with extreme sensitivity and specificity (as indicated by high true positive and low false positive rates), confirming previous observations in SAGM. ${ }^{25} \mathrm{~A}$ simplified overview of purine catabolism and oxidation intermediates is outlined. Structures are provided for adenine (top right) and hypoxanthine (bottom right).

$\mu \mathrm{M}$ and $800 \mu \mathrm{M}$, respectively, consistent with previous studies using other storage solutions (e.g. SAGM). ${ }^{16,31}$ Quantitative hypoxanthine levels also allow discrimination between three metabolic phases of the RBC storage lesion, as previously determined by receiver operating characteristic (ROC) curves for RBC stored in SAGM. ${ }^{28}$ Here we show similar results for RBC stored in AS-3 (Figure 1B) on the basis of absolute quantitative measurements, confirming and improving on the previous relative quantitative measurements (i.e., arbitrary units). ${ }^{28}$

Deamination of RBC purines (Figure 1C) by purine deaminases, such as AMPD, was initially described in the 1930s. ${ }^{43}$ More recent studies focused on the role of oxidative stress in activating $\mathrm{AMPD} 3$, the RBC-specific isoform. ${ }^{44}$ Consistent with observations in humans, hypoxanthine levels were reported to increase in stored C57BL/6J mouse RBC samples, without separation of cells and supernatants; ${ }^{45}$ here we confirmed and extended this observation by separately analyzing stored mouse RBC and supernatants (Figure 2A). Interestingly, in contrast to the human samples (Figure 1A), supernatants of mouse $\mathrm{RBC}$ stored in AS-3 contained less hypoxanthine at the end of storage as compared to RBC cytosol (Figure 1B).

Despite increased understanding of the storage lesion, ${ }^{2}$ it remains controversial whether the (metabolic) storage lesion in vitro holds any clinical relevance. ${ }^{3}$ PTR is - according to regulations of the US Food and Drug Administration - a standard measure of RBC storage quality with potential clinical relevance, in that the capacity to circulate in the recipient's bloodstream is a necessary (but not sufficient) requirement for $\mathrm{RBC}$ to function in vivo. We, therefore, performed $24 \mathrm{~h}$ PTR studies in mice and humans, and compared these results with hypoxanthine levels (Figure 2B). To this end, stored RBC from 79 mice of
14 different strains were assayed for hypoxanthine levels and PTR studies were completed following transfusion into C57BL6 recipients. As a result, a significant negative correlation was found (median of all strains $P<0.0001 ; \mathrm{r}=$ -0.87 Spearman; $r=-0.88$ for 8 out of 14 strains) (Figure 2C). Similarly, pre-transfusion hypoxanthine levels in stored human RBC from healthy volunteers ( $n=52$, transfused RBC from week 1-6) negatively correlated with PTR in the autologous transfusion setting $(P=0.0018, \mathrm{r}=-0.43$ Spearman) (Figure 2D).

\section{Oxygen saturation affects purine deamination in vivo and ex vivo}

Refrigerated RBC storage promotes oxidative stress, which is mitigated, in part, by decreasing $\mathrm{SO}_{2}$ during hypoxic storage. ' Strikingly, $\mathrm{SO}_{2}$ levels in 977 freshly donated units assayed within $8 \mathrm{~h}$ of donation and standard processing were widely distributed on storage day 1 (Online Supplementary Figure S1), ranging from 5\% to 95\%. This indicates that $\mathrm{SO}_{2}$ at donation is currently an uncontrolled variable in the donor population with the potential to affect RBC purine deamination. We, therefore, performed a series of experiments to determine whether exposure to hypoxia or hyperoxia in vitro or in vivo affected purine deamination and hypoxanthine accumulation in human and mouse RBC. Notably, exposure of healthy volunteers (and good acclimatizers) to high altitude hypoxia (>5000 $\mathrm{m}$ for up to 7 days) (Figure 3A) led to significant decreases in RBC hypoxanthine levels, even by $3 \mathrm{~h}$ (ALT1am, $P=0.02$ ) and $8 \mathrm{~h}$ (ALT1pm, $P=0.0002$ ) after ascent, which were even greater after 7 days at high altitude $\left(P=8.9 \times 10^{-5}\right)$ (Figure $\left.3 \mathrm{~B}\right)$. Similarly, exposure of C57BL/6 mice $(n=6)$ to hypobaric hypoxia $\left(8 \% \mathrm{O}_{2}\right.$ for up to $8 \mathrm{~h})$ led to significant $(P<0.01)$ decreases in $\mathrm{RBC}$ hypo- 


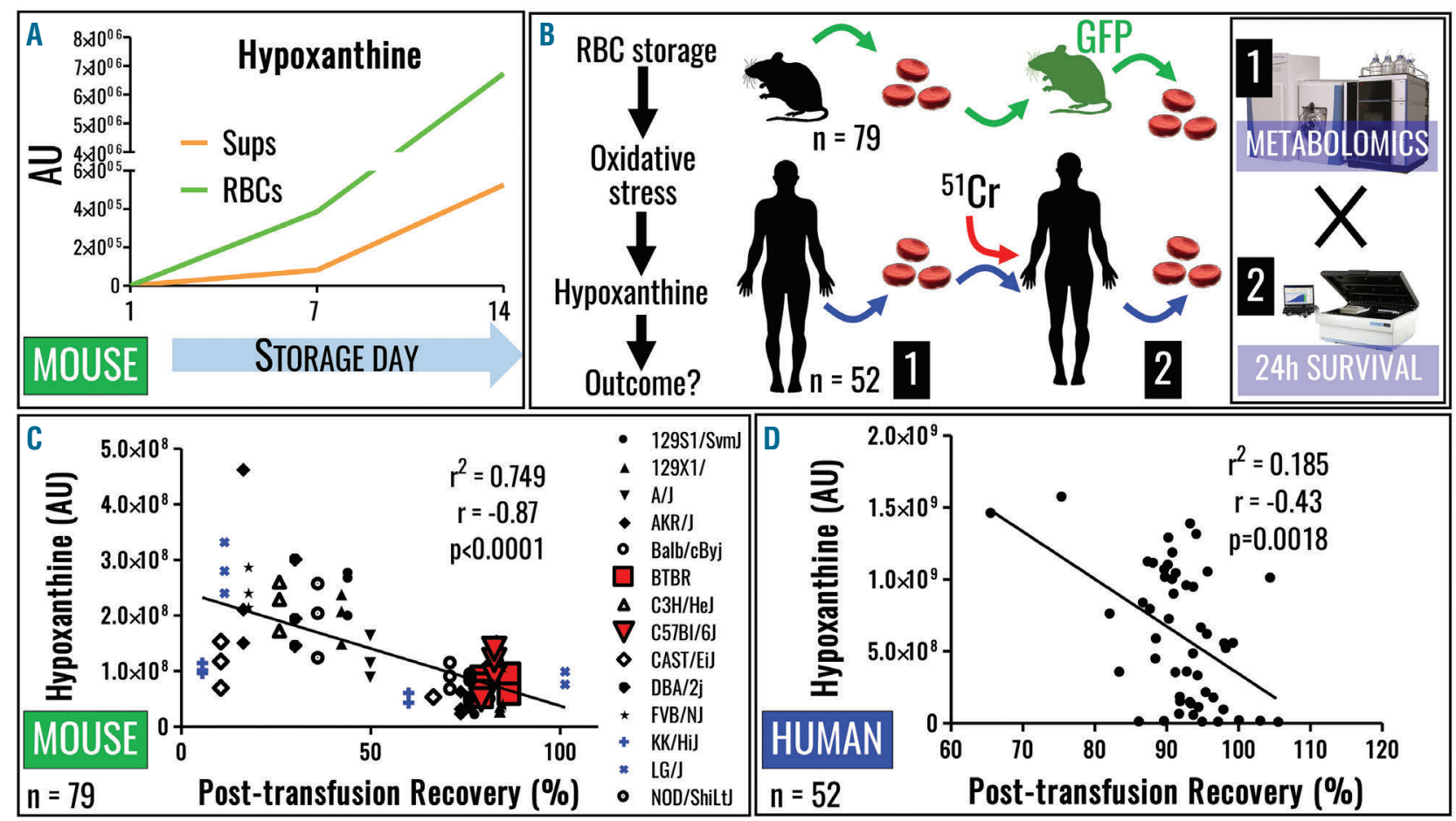

Figure 2. Hypoxanthine negatively correlates with post-trasfusion recovery of using mouse and human red blood cells. (A) Hypoxanthine accumulation is observed in stored C57BL/6J mouse RBC. (B) Transfusion into GFP-RBC mouse recipients (sorting of fluorescence negative RBC) or ${ }^{51} \mathrm{Cr}$ labeling of human RBC was performed to determine $24 \mathrm{~h}$ PTR in mice $(n=79)$ and human volunteers $(n=52)$, whereas paired samples were used to determine hypoxanthine levels. (C, D) Negative correlations were observed for mouse (C) and human RBC hypoxanthine levels and $24 \mathrm{~h}$ PTR. Linear and quadratic Spearman correlations as well as their levels of significance are shown for mouse (median across strains) and (D) human data.
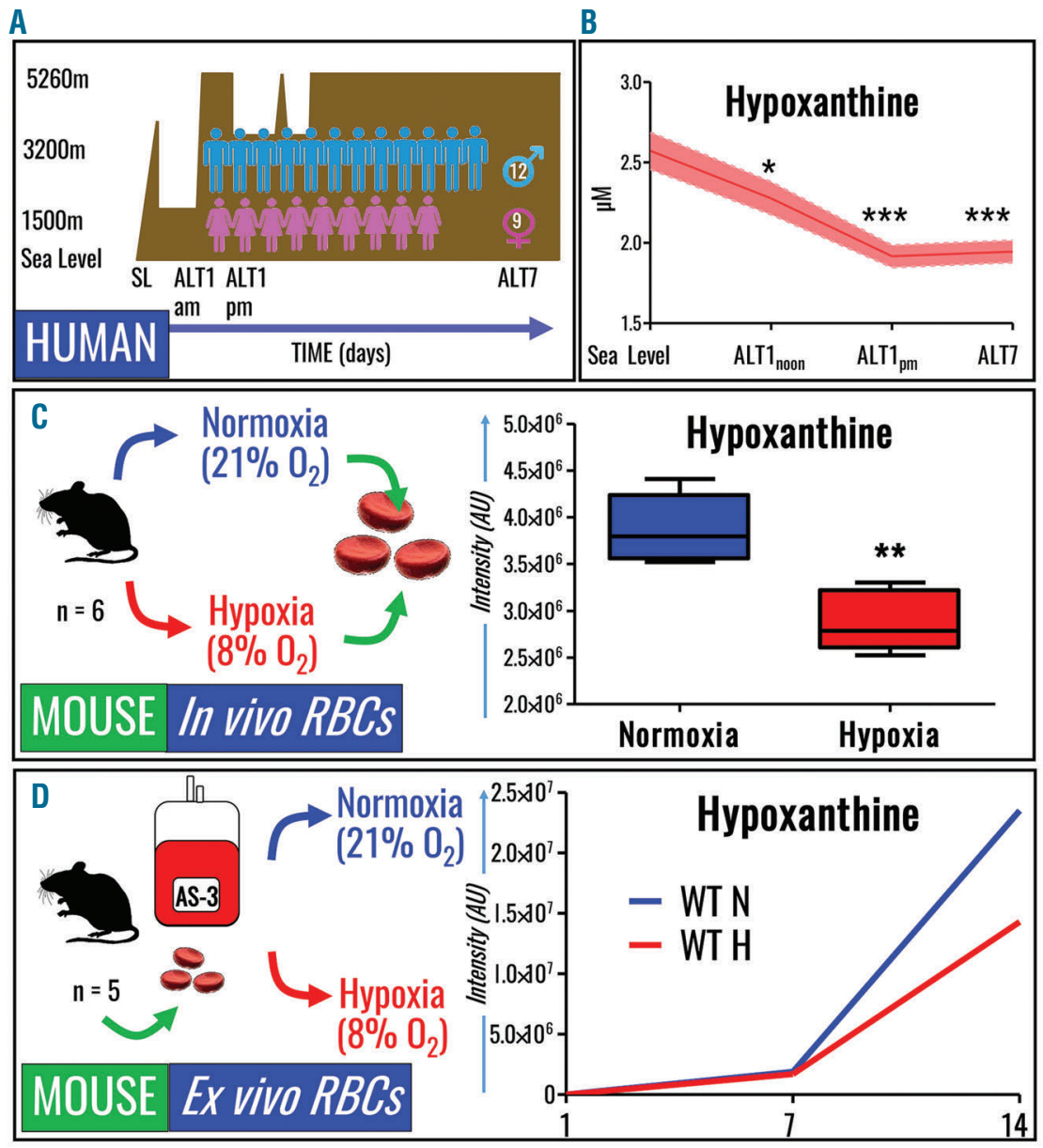

Figure 3. Hypoxanthine levels decrease in human and mouse red blood cells exposed to hypoxia in vivo and in vitro. (A) RBC were collected from 21 healthy volunteers at sea level (SL) and within 3 or $>8 \mathrm{~h}$ after exposure to high altitude hypoxia on day 1 (ALT1 am and pm, respectively), and at 7 days (ALT7) after exposure to high altitude $(>5000 \mathrm{~m})$ hypoxia. (B) Hypoxanthine levels decreased significantly in human RBC within hours of exposure to high altitude hypoxia ( $\mathrm{x}$ axis labels consistent with description of panel A). (C) C57BL/6J mice $(n=6)$ were exposed to normoxia or $8 \%$ oxygen for $3 \mathrm{~h}$, resulting in decreases in RBC levels of hypoxanthine, a phenomenon accompanied by decreased IMP and increased AMP/IMP ratios (Online Supplementary Figure S1). (D) RBC were collected from C57BL/6J mice prior to in vitro storage in AS-3 for up to 2 weeks under normoxic or hypoxic conditions, resulting in decreased hypoxanthine accumulation. 
xanthine levels (Figure 3C), while boosting AMP/IMP ratios (Online Supplementary Figure S2). In addition, when mouse RBC ( $n=5)$ were stored for up to 2 weeks under normoxic or hypoxic $\left(8 \% \mathrm{O}_{2}\right)$ conditions, lower hypoxanthine levels were seen with hypoxia (Figure $3 \mathrm{D}$ ). Analogously, human $\mathrm{RBC}(\mathrm{n}=4)$ were stored under controlled $\mathrm{SO}_{2}$ conditions, ranging from hyperoxia $\left(\mathrm{SO}_{2}>95 \%\right)$, to normoxia (no $\mathrm{SO}_{2}$ control), to hypoxia $\left(\mathrm{SO}_{2}=20 \%, 10 \%, 5 \%\right.$ or $<3 \%$ ) (Figure 4A). Although all conditions induced storagedependent increases in cellular and supernatant hypoxanthine levels, hypoxic RBC (independently of the degree of hypoxia) resulted in significantly $(P<0.01)$ lower levels of intracellular hypoxanthine from storage day 14 onwards (Figure 4B - left panel). Supernatant hypoxanthine levels were significantly lower in all hypoxic RBC after storage day 14 (Figure 4B - right panel). No significant effect of hyperoxia $\left(\mathrm{SO}_{2}>95 \%\right)$ was observed, except for higher than control $(P<0.05)$ supernatant hypoxanthine levels at storage day 7 , consistent with previous observations that $\mathrm{SO}_{2}$ can increase to $>95 \%$ by storage week 3 in normoxic (uncontrolled $\mathrm{SO}_{2}$ ) $\mathrm{RBC}$ with day $0 \mathrm{SO}_{2}>60 \%$ (though units in this study were agitated biweekly after sampling). ${ }^{9}$

Purine salvage and deamination in human and mouse red blood cells as a function of oxidative stress

In the previous section we showed that oxygen saturation affects hypoxanthine accumulation in human and mouse $\mathrm{RBC}$ in vitro and in vivo. Mechanistically, hypoxia-

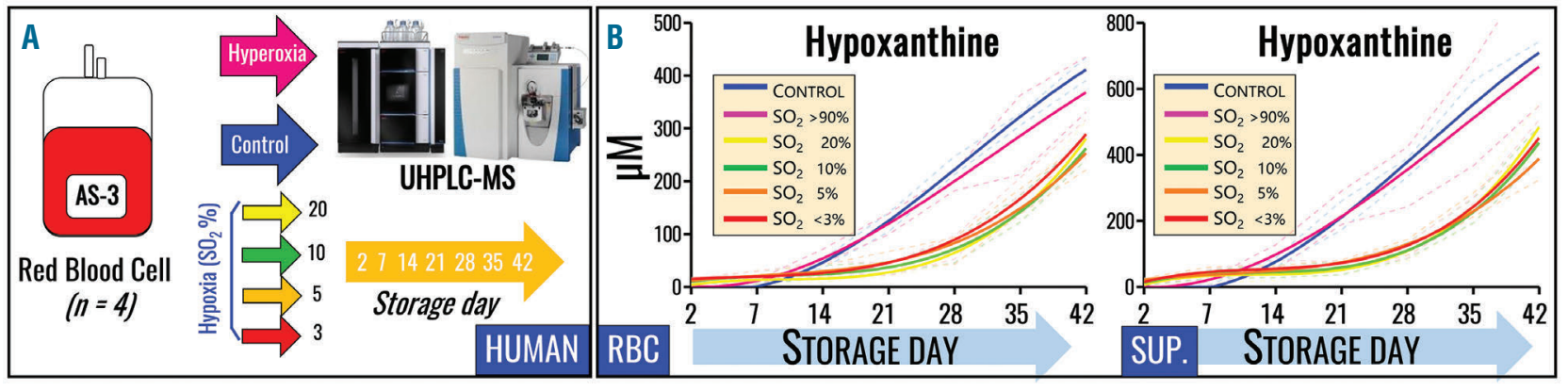

Figure 4. Effects of oxygene saturation on hypoxanthine accumulation during refrigerated storage. (A) Human RBC were donated by healthy volunteers ( $\mathrm{n}=4$ ) prior to refrigerated storage in AS-3 under control (normoxic), hyperoxic $\left(\mathrm{SO}_{2}>95 \%\right)$, or hypoxic $\left(\mathrm{SO}_{2}=20 \%, 10 \%, 5 \%\right.$ or $<3 \%$ ) conditions for up to 42 days. (B) Hypoxanthine concentration was determined in cells and supernatants, with significant decreases in the presence of hypoxia. All data points shown on the $x$ axes were tested and interpolated with third order polynomial curves (not assuming linear evolution of hypoxanthine accumulation during storage) and medians \pm ranges ( $\mathrm{n}=4$ ) are shown (dark blue lines and light blue areas, respectively).

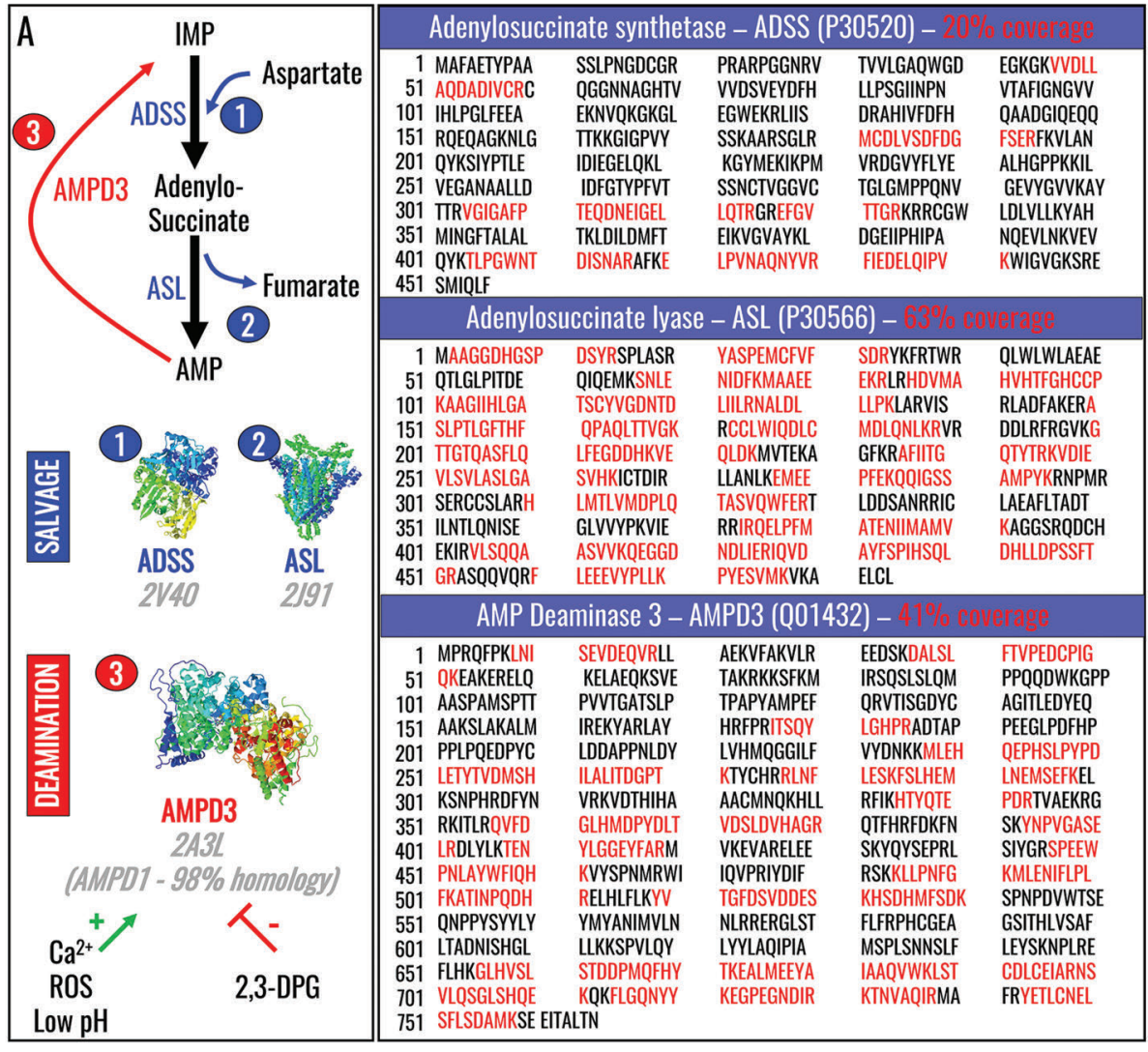

Figure 5. Purine salvage and deamination reactions. (A) Purine salvage and deamination reactions are catalyzed by adenylosuccinate synthase (ADSS - 1) and adenylosuccinate lyase (ASL - 2), and by adenosine monophosphate deaminase (AMPD3 - 3), respectively. (B) Deep proteomic analyses identified ADSS, ASL and AMPD3 in human RBC, in contrast to prior studies indicating the absence of ADSS in mature human $\mathrm{RBC}^{42}$ 
dependent decreases in purine deamination may be explained either by decreases in AMPD3 activity or increases in salvage reactions, including those catalyzed by adenylosuccinate synthetase (ADSS) and adenylosuccinate lyase (ASL) (Figure 5A). Deep proteomic analyses of RBC from healthy donor volunteers identified AMPD3, ASL (also confirmed by Western blot in mouse $\mathrm{RBC}$ ) (Online Supplementary Figure S3), and traces of ADSS in human RBC (sequence coverage shown in Figure $5 B$ ), despite classic literature indicating the absence of ADSS in mature human RBC. ${ }^{46}$ Mascot scores for peptide hits for each one of these proteins are reported in Online Supplementary Table S1.

AMPD3 activity is stimulated by oxidative stress, ${ }^{44}$ an observation supported herein by metabolomics evidence demonstrating increased inosine monophosphate (IMP) and hypoxanthine, and decreased AMP and AMP/IMP ratios, in human $\mathrm{RBC}$ exposed to hydrogen peroxide-generating xanthine dehydrogenase/oxidase $(\mathrm{XDH})$ in the presence of hypoxanthine (at levels similar to those observed in RBC supernatants at the end of storage; i.e., the high $\mu \mathrm{M}$ to low $\mathrm{mM}$ range) (Figure $6 \mathrm{~A}$ ).

G6PD is the rate-limiting enzyme of the pentose phosphate pathway (PPP), which is essential for $\mathrm{RBC}$ redox homeostasis because it generates reducing cofactors (i.e., $\mathrm{NADPH}$ ) to recycle oxidized glutathione back to its reduced form. Thus, we hypothesized that refrigerated storage of RBC from G6PD-deficient subjects increases oxidative stress and would result in increased hypoxan- thine levels. In our hands, refrigerated storage of $\mathrm{RBC}$ from G6PD-deficient volunteers ( $\mathrm{n}=6$; Mediterranean variant, $<10 \%$ residual activity, non-hemolytic) demonstrated significant increases in hypoxanthine levels in comparison to controls, beginning after storage day 14 (Figure 6B).

\section{Hypoxia prevents hypoxanthine accumulation by deregulating adenosine monophosphate deamination rather than by promoting salvage reactions}

Based on our observations, oxidative stress promoted purine deamination and hypoxanthine accumulation in RBC after: (i) incubation with xanthine dehydrogenase in the presence of hypoxanthine, and (ii) refrigerated storage, particularly in the case of G6PD-deficient donors. Conversely, hypoxia in vivo and in vitro has a beneficial role in mice and humans, by preventing purine deamination and hypoxanthine accumulation. Enzymes involved in salvage reactions (ADSS and ASL) and purine deamination (AMPD3) have been identified in mature RBC via deep proteomic measurements, suggesting that either pathway may be susceptible to oxidative stress and/or hypoxia. To expand on these steady state observations to test actual fluxes through these pathways, human $\mathrm{RBC}$ were incubated with ${ }^{13} \mathrm{C}_{1}$-aspartate for $6 \mathrm{~h}$ to determine the rate of "heavy" fumarate accumulation through salvage reactions (Figure 7A). Accumulation of isotopologue +1 of fumarate from aspartate may be alternatively explained by fumarate hydratase and malate dehydrogenase activity on
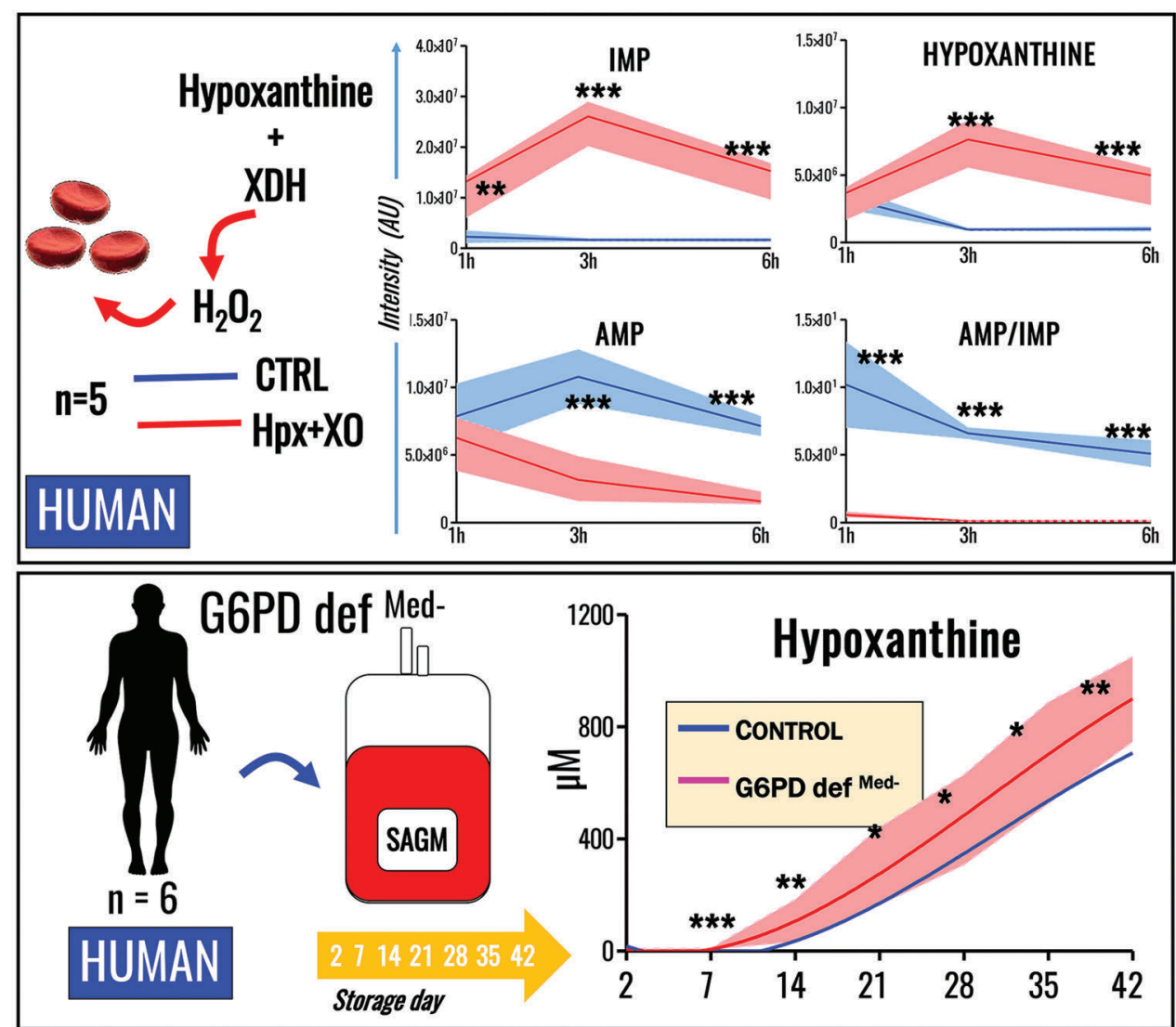

Figure 6. Oxidative injury or impairment of the antioxidant capacity in glucose-6-phosphate dehydrogenase-deficient red blood cells enhances purine deamination. (A) Exposure of human RBC to prooxidant treatment with hypoxanthine plus xanthine dehydrogenase $(\mathrm{XDH})$ for up to $6 \mathrm{~h}$ enhances AMP deamination to IMP accompanied by accumulation of hypoxanthine. (B) Human RBC from G6PD-deficient donors (Mediterranean variant, $<10 \%$ residual activity of the enzyme) were stored for up to 42 days, showing significantly higher levels of hypoxanthine throughout the whole storage period (analyses were performed on whole trans fusates: cells + supernatants) (median + ranges for RBC from G6PD donors are plotted as a solid red line and light red area, respectively). In (B), all data points shown on the $x$ axis were tested and interpolated with third order polynomial curves (not assuming linear evolution of hypoxanthine accumulation during storage). 
oxaloacetate derived from transamination of aspartate, reactions possible in mature erythrocytes. ${ }^{47}$ Although heavy aspartate consumption was faster in hypoxic RBC, the rates of ${ }^{13} \mathrm{C}$-fumarate generation were not sufficiently higher than in normoxic controls to explain the observed increases in AMP/IMP ratios in hypoxic RBC (e.g., only $\sim 26-30 \%$ of fumarate was labeled at $6 \mathrm{~h}$ after incubation with heavy aspartate in both normoxic and hypoxic groups). In parallel, human RBC were incubated for $6 \mathrm{~h}$ with ${ }^{13} \mathrm{C}_{5}$-adenosine to determine the rate of purine deamination (Figure 7B), demonstrating significantly decreased rates of IMP generation in hypoxic RBC. Analogously, refrigerated storage of mouse RBC under hypoxic conditions prevented purine deamination, as demonstrated by ${ }^{13} \mathrm{C}_{5}$-adenosine tracing experiments (Figure $7 \mathrm{C}$ ). Finally, incubating mouse RBC with deoxycoformycin, an adenosine and AMPD inhibitor, mimicked the hypoxic phenotype in terms of purine deamination and increased AMP/IMP ratios (Figure 7D). Thus, these results suggest that hypoxanthine accumulation, in this context, is primarily due to purine deamination.

\section{Discussion}

Recently, we and others identified hypoxanthine $e^{19,28}$ as a potential metabolic marker of the RBC storage lesion with potential clinical implications. Indeed, circulating hypoxanthine can be readily converted to xanthine and urate by reactions that generate hydrogen peroxide ${ }^{48}$ For exam-
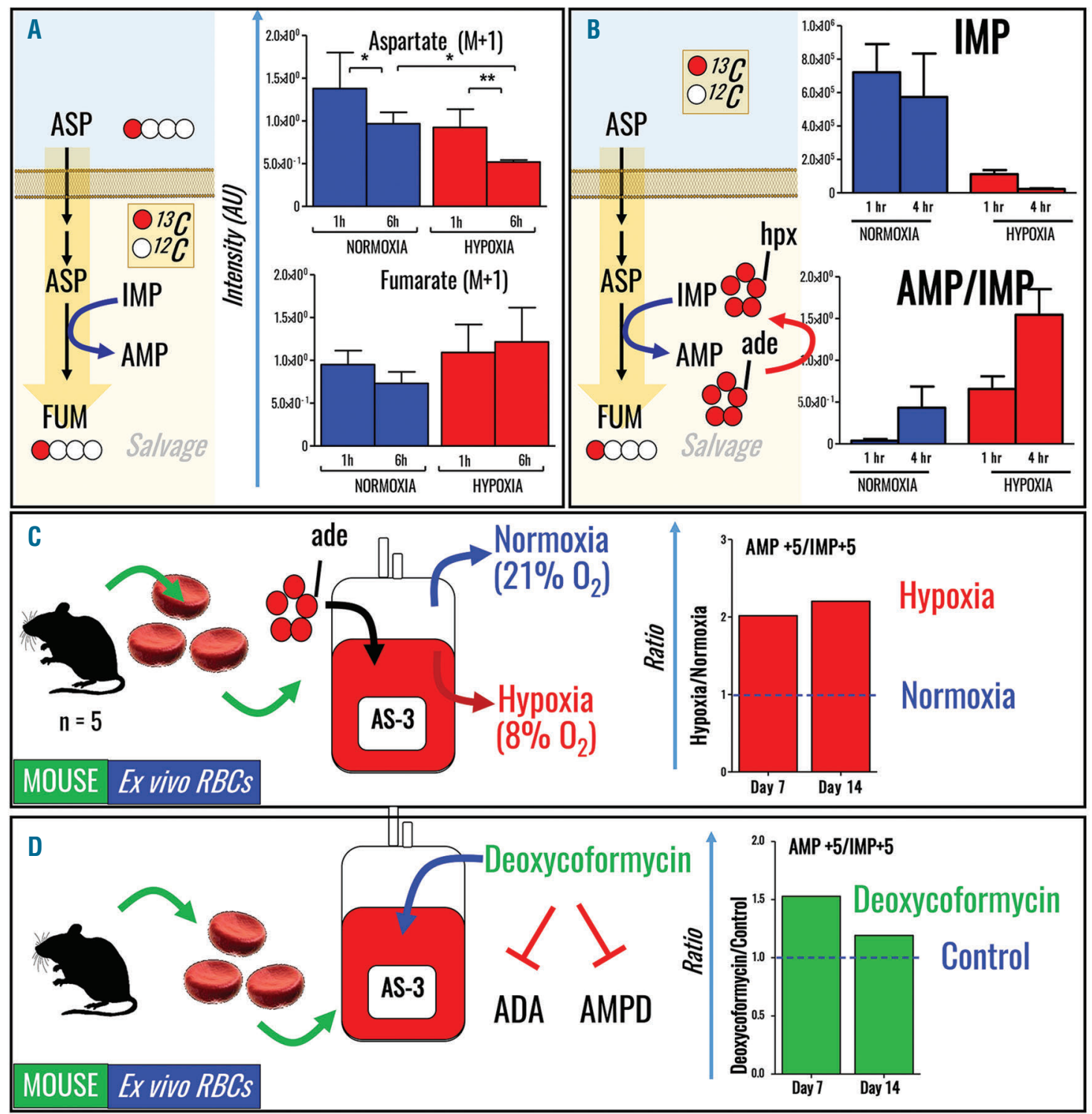

Figure 7. Tracing experiments reveal hypoxic inhibition of purine deamination, rather than hypoxic increases in purine salvage. (A, B) Human RBC were incubated with $(A){ }^{13} C_{1}$-aspartate to determine the rate of fumarate generation (salvage) and with $(B){ }^{13} C_{5}$-adenosine to determine the rate of $A M P$ deamination. $(C){ }^{13} C_{5}$-adenosine tracing experiments demonstrated that in vitro storage of mouse RBC under hypoxic conditions prevents purine deamination. (D) Incubation of mouse RBC with deoxycoformycin, an adenosine and AMP deaminase inhibitor, increases AMP/IMP ratios. 
ple, ischemic accumulation of plasma hypoxanthine can fuel pro-oxidant reactions following reperfusion. ${ }^{49}$ For this reason, rejuvenated RBC need to be washed prior to transfusion to avoid excess infusion of inosine and its metabolic product, hypoxanthine. ${ }^{50}$ By quantifying RBC and supernatant hypoxanthine levels during storage in AS-3, we confirmed and extended previous observations made in $\mathrm{RBC}$ stored in $\mathrm{SAGM}^{16,19}$ concerning the role of this metabolite as a reliable marker of the storage lesion. Thus, we correlated pre-transfusion in vitro hypoxanthine levels in mouse and human RBC with PTR determinations in vivo; the latter is one of the key US Food and Drug Administration's criteria for approving novel RBC storage methods. ${ }^{24}$ Because RBC must circulate to perform their therapeutic function, we believe that - provided the evidence in mice and preliminary findings in humans reported here are further prospectively validated - this observation may support the clinical relevance of hypoxanthine as a potential predictor of transfusion outcomes. In addition, despite intrinsic metabolic differences in purine catabolism between rodents and humans (e.g., the former express functional uricase ${ }^{51}$ which may contribute to explaining the minor inter-species difference in hypoxanthine levels in cells and supernatants observed here), hypoxanthine levels in vitro correlated negatively with PTR in vivo in both mice and - to a lesser, albeit significant, extent - humans, further documenting the relevance of animal models in transfusion medicine research. ${ }^{52}$

During the last few decades, retrospective clinical studies ${ }^{53}$ coupled with improved mechanistic understanding of the RBC storage lesion, ${ }^{2}$ have prompted the field of transfusion medicine to question the safety and efficacy of stored RBC. Recent prospective clinical trial evidence reassured the field about the non-inferiority of the current standard of care when compared to transfusion of fresh units. ${ }^{20}$ Nonetheless, transfusion of RBC stored for more than 5 weeks significantly increases circulating non-transferrin-bound iron levels, ${ }^{21}$ potentially increasing the risk of complications in certain categories of recipients. ${ }^{22}$ Omics markers of the storage lesion were recently identified, ${ }^{28,29}$ with the goal of benchmarking novel strategies or additive solutions to improve RBC storage quality. However, the disconnect between the well-established metabolic storage abnormalities and the reassuring clinical trial evidence prompted the field to question the relevance of metabolic markers of RBC storage age with respect to transfusion outcomes, a critical issue extensively discussed by many key opinion leaders. ${ }^{54}$ For example, over the past five decades, biochemical studies clearly documented that impairment of RBC energy and redox homeostasis (especially regarding levels of ATP and reduced glutathione) negatively affects $\mathrm{RBC}$ recovery in vivo, ${ }^{55}$ along with oxygen transport and delivery. Interestingly RBC levels of ATP and glutathione (as well as levels of at least 24 metabolites correlating with those of RBC ATP) are heritable traits, ${ }^{56}$ like hemolysis in vitro, although hemolysis does not correlate with pre-transfusion levels of these metabolites. ${ }^{57}$ Thus, it has been argued that these metabolites do not accurately predict transfusion outcomes likely because, as historically appreciated for 2,3-DPG ${ }^{58,59}$ their levels are restored by $50 \%$ within the first $4 \mathrm{~h}$ after transfusion and return to normal by $72 \mathrm{~h}$. However, these rates may not be sufficient to restore optimal tissue oxygenation in critically ill recipients who require massive transfusion, even though tissue oxygenation and systemic acidosis are effi- ciently restored by current resuscitation strategies.

Mechanistically (Figure 8), we propose that refrigerated RBC storage promotes, among many cascades of events, ${ }^{2-5}$ the activation of AMPD3 (the RBC-specific isoform of AMPD, and the only one identified here using deep proteomics). AMPD3 can be activated by increases in intracellular $\mathrm{ROS}^{44}$ and calcium, ${ }^{60,61}$ along with decreases in intracellular $\mathrm{pH}^{6,62,63}$ in contrast, increases in 2,3-DPG downregulate AMPD3 activity ${ }^{63}$ Notably, refrigerated RBC storage, especially after storage day 14, leads to increases in ROS and intracellular calcium, and decreases in $\mathrm{pH}$ and 2,3DPG ${ }^{2,6}$ all of which can combine to activate AMPD3, as our steady state and metabolic flux results suggest. This concept is supported by the positive effects of hypoxic storage, including decreased purine deamination leading to decreased hypoxanthine levels. Interestingly, AMPD3 activation shortens RBC lifespan ${ }^{64}$ by converting AMP into IMP, thereby contributing to adenine nucleotide dysregulation in RBC from normal individuals and those with sickle cell anemia. Conversely, the human enzymopathy of AMPD3 deficiency does not produce any relevant phenotype except for inducing increased levels of RBC ATP and ADP, thereby promoting a hypometabolic state with decreased hemoglobin oxygen affinity ${ }^{65,66}$

Being at the crossroads between energy generation and redox metabolism, AMPD3 represents an ideal target for modulating $\mathrm{RBC}$ metabolism during refrigerated storage. Here we show that oxygen saturation and oxidative stress during refrigerated storage modulate purine deamination in human and mouse RBC in vivo and in vitro. This is relevant because of the unexpected widespread distribution of oxygen saturation of freshly donated human RBC within $8 \mathrm{~h}$ after donation and routine normoxic processing, suggesting that oxygen levels may represent a relevant variable that can be controlled to enhance the uniformity of stored blood ${ }^{35}$ In addition, RBC from human G6PD-deficient donors - characterized by increased basal and storage-dependent oxidative stress ${ }^{67}$ - produce higher levels of hypoxanthine during refrigerated storage as compared to

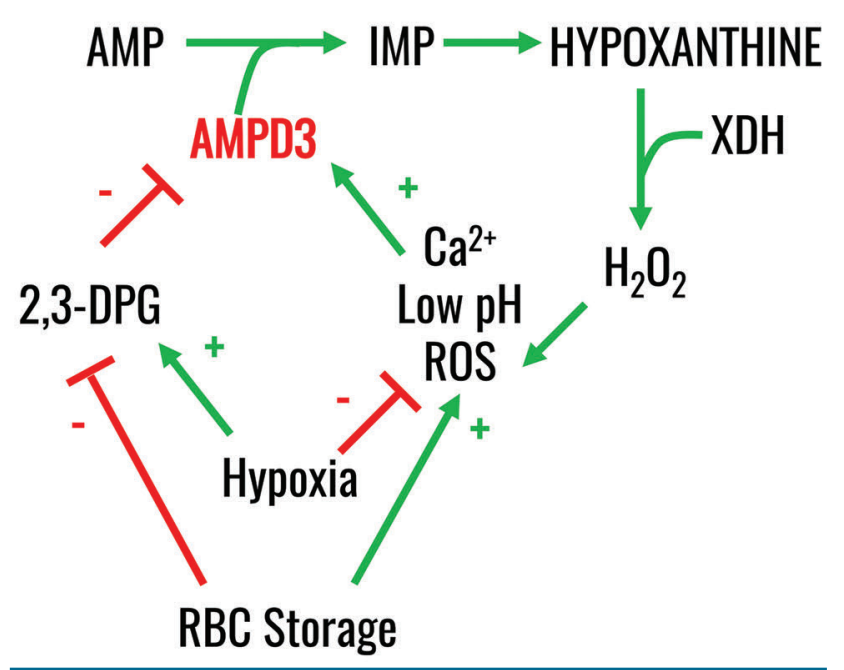

Figure 8. Proposed mechanism of the effect of hypoxia on the purine salvage pathway. RBC storage or oxidative stress promotes activation of RBC AMPD3, which in turn catalyzes purine deamination. This phenomenon is in part counteracted by $\mathrm{RBC}$ exposure to hypoxia in vivo or ex vivo, which phenocopies the pharmacological inhibition of purine deaminases. 
controls. G6PD activity in stored human RBC was reported to decline in SAGM additives, ${ }^{67,68}$ though no notable changes in G6PD activity ${ }^{69}$ or PPP fluxes, as determined by $\left[1,2,3^{13} \mathrm{C}_{3}\right]$ glucose tracing experiments, ${ }^{9}$ were noted in AS-3 stored RBC. In addition, although human G6PD activity is linked to gender (i.e., the G6PD gene is on chromosome $\mathrm{X})$ and decreases with age, ${ }^{70}$ correlations between donor age or gender with transfusion outcomes are controversial. ${ }^{71,72}$ Notably, diamide treatment of G6PD-deficient $\mathrm{RBC}$ induced increases in AMP, IMP and hypoxanthine, ${ }^{73}$ consistent with our results with human G6PD-deficient RBC. ${ }^{7}$ In human G6PD-deficient RBC, increases in AMP were explained by the inability to reduce oxidized glutathione using PPP-derived NADPH, which, in turn, required increases in ATP-consuming de novo GSH synthesis. $^{73}$

Tracing experiments with ${ }^{13} \mathrm{C}_{1}$-aspartate and ${ }^{13} \mathrm{C}_{5}$-adenosine show that hypoxic storage of $\mathrm{RBC}$ induces decreased AMPD3 activity, rather than increased purine salvage. The deep proteomic analyses identified traces of ADSS and ASL, and residual activity of the salvage pathway in mature RBC, thereby extending prior findings. ${ }^{43,46}$ Pharmacological inhibition of purine deaminase activity in normoxic RBC using deoxycoformycin mimicked the benefits of hypoxic storage, suggesting that manipulating the purinergic signaling axis using novel storage additives may improve RBC storage quality by enhancing energy production and redox homeostasis, ${ }^{74}$ similar to the metabolic adaptations of RBC to high altitude hypoxia. ${ }^{75}$

Finally, it is worth noting that, historically, circulating hypoxanthine levels (not RBC levels) have been suggested as a marker of hypoxia. ${ }^{49}$ Plasma hypoxanthine, under hypoxic conditions (e.g. high altitude or ischemia), may affect the severity of the reperfusion injury during its conversion to urate, a reaction that simultaneously generates pro-oxidant hydrogen peroxide. ${ }^{49}$ Similarly, accumulation of dicarboxylates and excess activation of salvage reactions in response to constrained oxygen availability were identified as mediators of oxidative reperfusion injury in mitochondria-proficient cells. ${ }^{76}$ The apparent disconnect between the present findings and the literature is reconciled by the observation that hypoxanthine levels in mitochondria-free RBC are not necessarily related to plasma levels of this metabolite (especially in the platelet and white blood cell-filtered environment of a packed RBC unit); for example, this is observed in underwater mammals, such as dolphins, which are constantly exposed to prolonged periods of hypoxia. ${ }^{77}$ Thus, hypoxanthine levels in isolated $\mathrm{RBC}$ (as in stored RBC units), or in circulating $\mathrm{RBC}$ after exposure to high altitude, may not directly correlate with plasma hypoxanthine levels.

\section{Conclusion}

Hypoxanthine is a marker of the RBC storage lesion in mice and humans in vitro; in addition, it correlates with PTR results in vivo in mice and, preliminarily, in humans though additional validation in larger cohorts including poor "recoverers" will be necessary. Storage-dependent increases in cytosolic and supernatant purine deamination during human and mouse RBC storage are ameliorated by hypoxia. In addition, oxidative stress, induced by exogenously added pro-oxidants or in response to PPP shutdown in G6PD-deficient human RBC, promotes purine deamination and increases intracellular levels of hypoxanthine. Pharmacological inhibition and metabolomics experiments with stable isotope tracers suggest that decreased AMPD3 activity provides a potential mechanistic explanation of the benefits (i.e., decreased purine deamination) associated with RBC exposure to hypoxia in vivo and in vitro. Future studies are needed to investigate whether the correlation between pre-transfusion levels of hypoxanthine in vitro and PTR results in vivo could be explained by the role that this metabolite plays as a substrate for generating hydrogen peroxide in the circulatory system of the transfusion recipient.

\section{Acknowledgments}

Research reported in this publication was supported in part by funds from the National Blood Foundation Early Career grant 2016 (ADA), the Boettcher Webb-Waring Biomedical Research Award - Early Career grant (ADA), the University of Colorado Comprehensive Cancer Center Core Support (P30 CA04693417 to $\mathrm{KCH}$ and $A D A)$, and grants from the National Institutes of Health: P50GM049222, T32GM008315, and P50GM049222 (KCH, ADA), R01GM113838 (ADo), and R01HL115557 (SLS). The content is solely the responsibility of the authors and does not necessarily represent the official views of the National Institutes of Health.

\section{References}

1. Ellingson KD, Sapiano MR, Haass KA, et al. Continued decline in blood collection and transfusion in the United States-2015. Transfusion. 2017;57(Suppl 2):1588-1598.

2. D'Alessandro A, Kriebardis AG, Rinalducci $S$, et al. An update on red blood cell storage lesions, as gleaned through biochemistry and omics technologies. Transfusion. 2015;55(1):205-219.

3. Zimring JC. Widening our gaze of red blood storage haze: a role for metabolomics. Transfusion. 2015;55(6):1139-1142.

4. Hod EA, Zhang N, Sokol SA, et al. Transfusion of red blood cells after pro- longed storage produces harmful effects that are mediated by iron and inflammation. Blood. 2010;115(21):4284-4292.

5. Klein HG. The red cell storage lesion(s): of dogs and men. Blood Transfus. 2017;15 (2):107-111.

6. D'Alessandro A, D'Amici GM, Vaglio S Zolla L. Time-course investigation of SAGMstored leukocyte-filtered red bood cell concentrates: from metabolism to proteomics. Haematologica. 2012;97(1):107-115.

7. Bardyn M, Tissot J-D, Prudent M. Oxidative stress and antioxidant defenses during blood processing and storage of erythrocyte concentrates. Transfus Clin Biol. 2017. pii: S1246-7820(17)30489-5.

8. Wither M, Dzieciatkowska M, Nemkov T, et al. Hemoglobin oxidation at functional amino acid residues during routine storage of red blood cells. Transfusion. 2016;56(2): 421-426.

9. Reisz JA, Wither MJ, Dzieciatkowska M, et al. Oxidative modifications of glyceraldehyde 3-phosphate dehydrogenase regulate metabolic reprogramming of stored red blood cells. Blood. 2016;128(12):e32-42.

10. Harper VM, Oh JY, Stapley R, et al. Peroxiredoxin-2 recycling is inhibited during erythrocyte storage. Antioxid Redox Signal. 2015;22(4):294-307.

11. Rinalducci S, D'Amici GM, Blasi B, et al. Peroxiredoxin-2 as a candidate biomarker to test oxidative stress levels of stored red blood cells under blood bank conditions. 
Hypoxanthine in stored RBC

Transfusion. 2011;51(7):1439-1449.

12. Eu X, Felcyn JR, Zimring JC. Bioactive lipids are generated to micromolar levels during $\mathrm{RBC}$ storage, even in leukoreduced units. Blood. 2015;126(23):2344-2344.

13. Silliman CC, Moore EE, Keller MR, et al. Identification of lipids that accumulate during the routine storage of prestorage leukoreduced red blood cells and cause acute lung injury. Transfusion. 2011;51(12):2549-2554.

14. D'Alessandro A, Nemkov T, Yoshida T, et al. Citrate metabolism in red blood cells stored in additive solution-3. Transfusion. 2017;57(2):325-336.

15. Sur C, Tariket S, Chou ML, et al. Duration of red blood cell storage and inflammatory marker generation. Blood Transfuse. 2017;15(2):145-152.

16. Bordbar A, Johnson PI, Paglia G, et al. Identified metabolic signature for assessing red blood cell unit quality is associated with endothelial damage markers and chinical outcomes. Transfusion. 2016;56(4): 852-862.

17. D'Alessandro A, Nemkov T, Keller M, et al. Routine storage of red blood cell (RBC) units in additive solution-3: a comprehensive investigation of the RBC metabolome. Transfusion. 2015;55(6):1155-1168.

18. Roback JD, Josephson CD, Waller EK, et al. Metabolomics of AS-1 RBC storage. Transfus Med Rev. 2014;28(2):41-55.

19. Pertinhez TA, Casali E, Lindner L, et al. Biochemical assessment of red blood cells during storage by $1 \mathrm{H}$ nuclear magnetic resonance spectroscopy. Identification of a biomarker of their level of protection against oxidative stress. Blood Transfuse. 2014;12 (4):548-556.

20. Belpulsi D, Spitalnik SL, Hod EA. The controversy over the age of blood: what do the clinical trials really teach us? Blood Transfuse. 2017;15(2):112-115.

21. Rapido F, Brittenham GM, Bandyopadhyay $S$, et al. Prolonged red cell storage before transfusion increases extravascular hemolysis. J. Chin. Invest. 2017;127(1):375-382.

22. Goel R, Johnson DJ, Scott AV, et al. Red blood cells stored 35 days or more are usocrated with adverse outcomes in high-risk patients. Transfusion. 2016;56(7):16901698.

23. Mays JA, Hess JR. Modelling the effects of blood component storage lesions on the quality of haemostatic resuscitation in massine transfusion for trauma. Blood Transfuse. 2017;15(2):153-157.

24. Dumont LJ, AuBuchon JP. Evaluation of proposed FDA criteria for the evaluation of radilabeled red cell recovery trials. Transfusion. 2008;48(6):1053-1060.

25. Spitalnik SL, Triulzi D, Levine DV, et al. 2015 Proceedings of the National Heart, Lung, and Blood Institute's State of the Science in Transfusion Medicine symposum. Transfusion. 2015;55(9):2282-2290.

26. Hess JR, Rugs N, Knapp AD, et al. Successful storage of ABCs for 10 weeks in a new additive solution. Transfusion. 2000;40(8):10121016.

27. Yoshida T, Shevkoplyas SS. Anaerobic storage of red blood cells. Blood Transfuse. 2010;8(4):220-236.

28. Paglia G, D'Alessandro A, Rolfsson Ó, et al. Biomarkers defining the metabolic age of red blood cells during cold storage. Blood. 2016;128(13):e43-50.

29. D'Alessandro A, Nemkov T, Reisz J, et al. Omics markers of the red cell storage lesion and metabolic linkage. Blood Transfuse. 2017;15(2):137-144.
30. Bardyn M, Rappaz B, Jaferzadeh K, et al. Red blood cells ageing markers: a multiparametric analysis. Blood Transfuse. 2017;15(3):239-248.

31. Casali E, Beni P, Spisni A, Baricchi R, Pertinhez TA. Hypoxanthine: a new paradigm to interpret the origin of transfusion toxicity. Blood Transfuse. 2015;14(6):555556.

32. Gilson CR, Kraus TS, Hod EA, et al. A novel mouse model of red blood cell storage and posttransfusion in vive survival. Transfusion. 2009;49(8):1546-1553.

33. Goldman N, Chen M, Fujita $T$, et al. Adenosine A1 receptors mediate local antinociceptive effects of acupuncture. Nat Neurosci. 2010;13(7):883-888.

34. de Wolski K, Eu X, Dumont LJ, et al. Metabolic pathways that correlate with post-transfusion circulation of stored murine red blood cells. Haematological. 2016;101(5): 578-586.

35. Yoshida T, Blair A, D'Alessandro A, et al. Enhancing uniformity and overall quality of red cell concentrate with anaerobic storage. Blood Transfuse. 2017;15(2):172-181.

36. D'Alessandro A, Nemkov T, Sun K, et al. AltitudeOmics: red blood cell metabolic adaptation to high altitude hypoxia. J. Proteome Res. 2016;15(10):3883-3895.

37. Baskurt OK, Temiz A, Meiselman HJ. Effect of superoxide anions on red blood cell rheologic properties. Free Radic Biol Med. 1998;24(1):102-110.

38. D'Alessandro A, Dzieciatkowska M, Nemkov T, Hansen KC. Red blood cell proteomics update: is there more to discover? Blood Transfuse. 2017;15(2):182-187.

39. Dzieciatkowska M, Hill R, Hansen KC. GeLC-MS/MS analysis of complex protein mixtures. Methods Mol Biol. 2014;1156:5366

40. Beth TS, Francavilla C, Olsen JV. Off-line high -pH reversed-phase fractionation for indepth phosphoproteomics. J Proteome Res. 2014;13(12):6176-6186

41. Nemkov T, Hansen KC, Dumont LJ, D'Alessandro A. Metabolomics in transfusion medicine. Transfusion. 2016;56(4):980993.

42. Nemkov T, Hansen KC, D'Alessandro A. A three-minute method for high-throughput quantitative metabolomics and quantitative tracing experiments of central carbon and nitrogen pathways. Rapid Commun Mass Spectrom. 2017;31(8):663-673.

43. Filer J, Worthington F. The catabolism of the purine nucleotides: I. the relation to glycolysis in the blood of the rabbit. J Biol Chem. 1938;123:655.

44. Tavazzi B, Amorini AM, Fazzina G, et al. Oxidative stress induces impairment of human erythrocyte energy metabolism through the oxygen radical-mediated direct activation of AMP-deaminase. J Biol Chem. 2001;276(51):48083-48092.

45. Zimring JC, Smith N, Stowell SR, et al. Strain-specific red blood cell storage, metalolism, and eicosanoid generation in a mouse model. Transfusion. 2014;54(1):137-148.

46. Low BA, Dorfman B-Z. Adenylosuccinase activity in human and rabbit erythrocyte lysates. J Biol Chem. 1970;245(12):30433046.

47. Bordbar A, Yurkovich JT, Paglia G, et al. Elucidating dynamic metabolic physiology through network integration of quantitative time-course metabolomics. Sci Rep. 2017;7:46249.

48. Kelley EE, Rho NKH, Hundley NJ, et al. Hydrogen peroxide is the major oxidant

product of xanthine oxidase. Free Radic Biol Med. 2010;48(4):493-498.

49. Saugstad OD. Hypoxanthine as an indicator of hypoxia: its role in health and disease through free radical production. Pediatr Res. 1988;23(2):143-150.

50. D'Alessandro A, Gray AD, Szczepiorkowski $\mathrm{ZM}$, et al. Red blood cell metabolic responsees to refrigerated storage, rejuvenation and frozen storage. Transfusion. 2017;57(4): 1019-1030.

51. Blair EM, Rawls KD, Dougherty BV, et al. Reconciled rat and human metabolic networks for comparative toxicogenomics and biomarker predictions. Nat Commun. 2017;8:14250

52. Zimring JC, Spitalnik SL. On the appropriate use and interpretation of animal models in transfusion medicine research. Transfusion. 2013;53(10):2334-2339.

53. Koch CG, Li L, Sessler DI, et al. Duration of red-cell storage and complications after cardiac surgery. N Angl J Med. 2008;358(12): 1229-1239.

54. D'Alessandro A, Liumbruno GM. Red blood cell storage and clinical outcomes: new insights. Blood Transfuse. 2017;15(2):101103

55. van Wijk R, van Soling WW. The energyless red blood cell is lost: erythrocyte enzyme abnormalities of glycolysis. Blood. 2005;106(13):4034-4042.

56. van 't Eve TJ, Wagner BA, Martin SM, et al. The heritability of metabolite concentratons in stored human red blood cells. Transfusion. 2014;54(8):2055-2063.

57. Van 't Eve TJ, Wagner BA, Martin SM, et al. The heritability of hemolysis in stored human red blood cells. Transfusion. 2015;55(6):1178-1185

58. Valeric CR, Hirsch NM. Restoration in vive of erythrocyte adenosine triphosphate, 2,3diphosphoglycerate, potassium ion, and sodium ion concentrations following the transfusion of acid-citrate-dextrose-stored human red blood cells. Transl Res. 1969;73 (5):722-733.

59. Butler E, Wood L. The in vive regeneration of red cell 2,3 diphosphoglyceric acid (DPG) after transfusion of stored blood. J Lab Chin. Med. 1969;74(2):300-304.

60. Mahnke DK, Sabina RL. Calcium activates erythrocyte AMP deaminase [isoform E (AMPD3)] through a protein-protein interactron between calmodulin and the N-termianal domain of the AMPD3 polypeptide. Biochemistry (Masc.). 2005;44(14):55515559.

61. Sabina RL, Wandersee NJ, Hillary CA. $\mathrm{Ca} 2+-\mathrm{CaM}$ activation of AMP deaminate contributes to adenine nucleotide dysregulaton and phosphatidylserine externalization in human sickle erythrocytes. $\mathrm{Br} \mathrm{J}$ Haematol. 2009;144(3):434-445.

62. Mahnke-Zizelman DK, Sabina RL. N-terminat sequence and distal histidine residues are responsible for $\mathrm{pH}$-regulated cytoplasmic membrane binding of human AMP deaminose isoform E. J Biol Chem. 2002;277 (45):42654-42662.

63. Dudley GA, Terjung RL. Influence of acidosis on AMP deaminase activity in contracting fast-twitch muscle. Am J Physiol. 1985;248(1 Pt 1):C43-50.

64. Hortle E, Nijagal B, Bauer DC, et al. Adenosine monophosphate deaminase 3 activation shortens erythrocyte half-life and provides malaria resistance in mice. Blood. 2016;128(9):1290-1301.

65. Daniels IS, Iii WGO, Neath V, Zhao Z, Lee CC. AMP deaminate 3 deficiency enhanced 
5 -AMP induction of hypometabolism. PLOS One. 2013;8(9): e75418.

66. O'Brien WG III, Berka V, Tsai A-L, Zhao Z, Lee CC. CD73 and AMPD3 deficiency enhance metabolic performance via erythrocyte ATP that decreases hemoglobin oxygen affinity. Sci. Rep. 2015;5:13147

67. Tzounakas VL, Kriebardis AG, Georgatzakou HT, et al. Glucose 6-phosphate dehydrogenase deficient subjects may be better "storers" than donors of red blood cells. Free Radic Biol Med. 2016;96:152-165.

68. Peters AL, van Bruggen R, de Korte D, Van Noorden CJ, Vlaar AP. Glucose-6-phosphate dehydrogenase activity decreases during storage of leukoreduced red blood cells. Transfusion. 2016;56(2):427-432.

69. Francis RO, Jhang J, Hendrickson JE, et al. Frequency of glucose-6-phosphate dehydrogenase-deficient red blood cell units in a metropolitan transfusion Transfusion. 2013;53(3):606-611.

70. Rodgers GP, Lichtman HC, Sheff MF. Red blood cell glucose-6-phosphate dehydrogenase activity in aged humans. J Am Geriatr Soc. 1983;31(1):8-11.

71. Chassé M, Tinmouth A, English SW, et al. Association of blood donor age and sex with recipient survival after red blood cell transfusion. JAMA Intern Med. 2016;176(9):13071314

72. Edgren G, Ullum $\mathrm{H}$, Rostgaard $\mathrm{K}$, et al Association of donor age and sex with survival of patients receiving transfusions. JAMA Intern Med. 2017;177(6):854-860

73. Tang $\mathrm{H}, \mathrm{Ho} \mathrm{H}$, Wu P, et al. Inability to maintain GSH pool in G6PD-deficient red cells causes futile AMPK sctivation and irreversible metabolic disturbance. Antioxid. Redox Signal. 2015;22(9):744-759.
74. Sun K, D'Alessandro A, Xia Y. Purinergic control of red blood cell metabolism: novel strategies to improve red cell storage quality. Blood Transfus. 2017;15(6):535-542.

75. Liu $\mathrm{H}$, Zhang Y, Wu H, et al. Beneficial role of erythrocyte adenosine A2B receptormediated AMP-activated protein kinase activation in high-altitude hypoxia. Circulation. 2016;134(5):405-421.

76. Chouchani ET, Pell VR, Gaude E, et al Ischaemic accumulation of succinate controls reperfusion injury through mitochondrial ROS. Nature. 2014;515(7527):431435.

77. López-Cruz RI, Crocker DE, Gaxiola-Robles $\mathrm{R}$, et al. Plasma hypoxanthine-guanine phosphoribosyl transferase activity in bottlenose dolphins contributes to avoiding accumulation of non-recyclable purines. Front Physiol. 2016;7:213 